\title{
INSTANTANEOUS FREQUENCY ESTIMATION USING LEVEL-CROSSING INFORMATION
}

\author{
S.Chandrasekhar ${ }^{1}$ and T.V.Sreenivas ${ }^{2}$ \\ Dept. of Electrical Communication Engg., \\ Indian Institute of Science, \\ Bangalore - 560 012, INDIA. \\ e-mail: ${ }^{1}$ schash@ protocol.ece.iisc.ernet.in \\ ${ }^{2}$ tvsree@ece.iisc.ernet.in
}

\begin{abstract}
In this paper, we discuss the problem of instantaneous frequency (IF) estimation of phase signals using their level-crossing (LC) instant information. We cast the problem to that of interpolating the instantaneous phase (IP), and hence finding the IF, from samples obtained at the level-crossing instants of the phase signal. These are inherently irregularly spaced and the problem essentially reduces to reconstructing a signal from the samples taken at irregularly sampled points for which we propose a line plus sum of sines' model. In the presence of noise, the temporal structure of the level-crossings can get distorted. To reduce the effects of noise, we use a short-time Fourier transform (STFT) based enhancement scheme. The performance of the proposed method is studied through Monte-Carlo simulations for a phase signal with composite IF for various SNRs. Different level-crossing based estimates are combined to obtain a new IF estimate. Simulation studies show that the estimates obtained using zero-crossing $(\mathrm{ZC})$ and other very low level values perform better than those obtained with higher level values.
\end{abstract}

\section{INTRODUCTION}

Most information-rich practical signals tend to be nonstationary random processes with time-varying spectral characteristics. Shorttime Fourier transform (STFT) and such other joint time-frequency (t-f) distributions (TFDs) attempt to describe the time-varying frequency and energy content of a nonstationary signal, frequency modulated (FM) signals being a typical example. Our present work focuses on using LC information for IF estimation and is motivated by several reasons. LC based approaches have been successfully employed in spectral estimation of stationary signals $[1,2]$. These approaches use either LC rate information or statistics of intervals between successive LCs. Extensions to nonstationary signals are discussed in [3] where a specific case, namely, $\mathrm{ZC}$ rate information was obtained on a short-time basis and was an ad-hoc extension of the analysis technique to deal with nonstationary signals under the implicit assumption of quasi-stationarity over the observation window. Hence, these are basically restricted to tracking mild frequency variations over long durations of time. If the assumption breaks down, we get unreliable frequency estimates. In this paper, we explore yet another source of information, namely, the LC instants. In particular, for frequency modulated signals, these can provide useful and alternate means of reconstructing the underlying FM.
Using the concept of time-warping, we find that the LCs essentially provide samples of the IP at irregularly spaced instants. The problem of IF estimation then reduces to reconstructing the IP using information obtained on an irregular geometry [4] followed by differentiation. Assuming continuous, positive, bandlimited IF, we propose a line plus sum of sines model for reconstructing the IF at any point within the observation window.

\section{PROBLEM FORMULATION}

Phase signals are real-valued signals with a constant instantaneous amplitude. Such signals contain all the information in the instantaneous phase or instantaneous frequency and are the basic elements of angle modulation schemes. They can be expressed as

$$
s(t)=A \sin (\phi(t)) .
$$

with $z_{s}(t)=A e^{j \phi(t)}$ being the corresponding analytic signal. It is desired to estimate the IF variation from the knowledge of the LC instants of the phase signal $s(t)$. We assume that the IF is positive. We also assume that the product of signal bandwidth $(B)$ and duration ( $\mathrm{T}$ ), often referred to as the $\mathrm{BT}$ product, is sufficient enough to observe any meaningful variation of the IF. The BT product of a signal can be thought of as a measure of "information-richness" in the signal. It makes little sense to characterize a signal by its IF if its BT product is too small since there will not be enough data to observe any meaningful variation.

For a sinusoid, the IF equals the Fourier frequency, and can be easily extracted from the LC information. Consider the case of a phase signal $s(t)=\sin (\phi(t))$ with arbitrary monotonic IP function, $\phi(t)$. If we denote the level, ' $l$ ' crossing instants as $t_{j}, j=0,1,2, \ldots$ then $\phi\left(t_{j}\right)=j \pi+(-1)^{j} \sin ^{-1}(l)$. Thus, partial information about the underlying IP and IF is contained in the LCs.

When plotted against $\phi(t)$, essentially warped-time, the phase signal appears as a constant frequency sine wave with the same number of LCs as in the time domain. The transformation that maps time to the warped-time is precisely the IP. Hence, if we map the nonuniformly spaced level ' $l$ ' crossing instants $t_{j}, j=$ $0,1,2, \ldots$ of the given phase signal to $j \pi+(-1)^{j} \sin ^{-1}(l)$ (taking phase unwrapping into account), we get partial information about the IP. Using this information, we need to obtain the IP at any point within the observation window. Finding the IF from the solution to IP then merely reduces to simple differentiation. Thus, the LC instants alone provide a novel means of estimating the IF. The total 
number of LC instants over the period of observation of the signal in both domains is the same. A monotone IP function (hence, positive IF) preserves a one-one relationship between time ' $t$ ' and warped-time, $\phi(t)$. It is the invariance of number of $L C s$ and their relative position over the observation interval that the algorithm makes use of, to estimate the IF.

\section{LINE PLUS SUM OF SINES MODEL}

In this section, we propose a novel model known as the line plus sum of sines model for reconstructing the IF from LC data.

Consider a finite window of the signal $s(t)$ which has a finite number of measured LC instants, $\left\{t_{j}, j=0,1, \ldots N\right\}$ with $t_{0}<$ $t_{1} \ldots<t_{N} \leq T$ within the analysis window, corresponding to a level, say ' $l$ '. For the purpose of estimation, let us assume that the $\mathrm{IP}, \phi(t)$ and the IF, $f_{i}(t)$ can be modeled as follows:

$$
\begin{aligned}
\phi(t) & =\alpha+\beta t+\sum_{k=1}^{p} \gamma_{k} \sin \left(k \omega_{o} t\right) \\
f_{i}(t) & =\frac{1}{2 \pi}\left[\beta+\omega_{0} \sum_{k=1}^{p} k \gamma_{k} \cos \left(k \omega_{o} t\right)\right]
\end{aligned}
$$

where $\omega_{0}=\frac{\pi}{T}$. It may be noted that this is a very natural model for phase signals with continuous, positive, bandlimited IF. While the sum of sines models the bandlimited part of IF, the line reflects the positive bias in IF.

Let us specify a cost-function $\mathcal{C}$ as a measure of approximation of the IP at the level ' $l$ ' - crossing instants by the approximating functions chosen as above.

$$
\mathcal{C}_{p}(\mathrm{c})=\sum_{j=0}^{N}\left|\phi^{l}\left(t_{j}\right)-\mathrm{c}^{T} e_{j}\right|^{2}
$$

where $e_{j}=\alpha+\beta t_{j}+\sum_{k=1}^{p} \gamma_{k} \sin \left(k \omega_{o} t_{j}\right)$ and $\mathrm{c}=\left[\alpha \beta \gamma_{1}\right.$ $\left.\gamma_{2} \ldots \gamma_{p}\right], \phi^{l}\left(t_{j}\right)=j \pi+(-1)^{j} \sin ^{-1}(l)$. Taking the gradient of $\mathcal{C}_{p}(\mathbf{c})$ with respect to $\mathrm{c}$ and setting it equal to zero will yield the optimum coefficient vector for the $p^{t h}$ order case, denoted by $\mathbf{c}^{\text {opt }}$. Thus, we can obtain,

$$
\left[\sum_{j=0}^{N} e_{j} e_{j}^{T}\right] \mathrm{c}^{\mathrm{opt}}=\sum_{j=0}^{N} \phi^{l}\left(t_{j}\right) e_{j}
$$

In matrix form, the same is expressed as

$$
\mathbf{R c}^{\text {opt }}=\tilde{\mathbf{e}}
$$

where $\mathbf{R}=\left[\sum_{j=0}^{N} e_{j} e_{j}^{T}\right], \tilde{\mathbf{e}}=\sum_{j=0}^{N} \phi^{l}\left(t_{j}\right) e_{j} . \mathbf{c}^{\text {opt }}$ can be solved through matrix inversion. Efficient numerical procedures exist to implement the same.

Let the estimate of IP obtained using level ' $l$ ' crossing information be denoted by $\widehat{\phi}^{t}(t)$. We get different estimates based on different LC information. These can be combined to yield a weighted-average

$$
\widehat{\phi}(t)=\frac{1}{\sum_{l=0}^{L-1} w_{l}} \sum_{i=0}^{L-1} w_{l} \widehat{\phi}^{l}(t)
$$

where $L$ is the total number of levels used and the weights $w_{l}$ are positive and normalized to add to unity.

\section{ENHANCEMENT OF NOISY SIGNAL}

In a practical situation, we work with sampled data and the data is often noisy. Noise can cause perturbation of signal LCs and/or introduce additional ones depending upon the SNR. At any instant, the spectral content of the signal occupies a specific region in frequency and 'out-of-band noise' may introduce additional LCs that can yield biased estimates. Since the signals under consideration are nonstationary, they have a time-varying spectral content. This implies that out-of-band noise occupies different portions of the spectrum at different instants of time and a stationary filter cannot separate the desired signal and noise. We describe a procedure that helps separate the two signals in a joint time-frequency domain which are otherwise inseparable in time or frequency alone. This is achieved by frequency selection in the STFT domain.

Consider the discrete-time phase signal $s[n]$ corrupted with additive white Gaussian noise $w[n]$. Let the noisy signal be denoted by $z[n]$ i.e., $z[n]=s[n]+w[n]$. Denote its STFT by $\mathcal{Z}\left[n, e^{i \omega}\right)^{1}$. We define $\widehat{s}[n]$, an enhanced version of $z[n]$ as

$$
\widehat{s}[n]=\frac{1}{2 \pi h(0)} \int_{-\pi}^{\pi} \mathcal{Z}\left[n, e^{i \omega}\right) \mathcal{H}(\omega-\widehat{\omega}[n]) e^{i \omega n} d \omega
$$

where $h[n]$ is the window function used in obtaining the STFT, $\widehat{\omega}[n]$ is the estimate of IF at the time instant $n$ obtained by peakpicking in the spectrogram t-f domain. $\mathcal{H}(\omega-\widehat{\omega}[n])$ is a filter centered at $\omega=\widehat{\omega}[n]$ and defined as follows:

$$
\mathcal{H}(\omega-\widehat{\omega}[n])= \begin{cases}1 & \text { for } \widehat{\omega}[n]-\frac{B}{2}<\omega<\widehat{\omega}[n]+\frac{B}{2} \\ 0 & \text { otherwise }\end{cases}
$$

where $B$ is chosen as the bandwidth between the first nulls and is determined by the STFT window shape and size. This is essentially a time-varying frequency domain selection applied to STFT. Such frequency domain windowing operations are frequently used in extracting robust features for speech recognition.

To illustrate the utility of the above procedure with an example, consider a phase signal with a composite IF, $f_{i}(n)=0.2+$ $0.05(\sin (0.12 n)+\sin (0.07 n)), 0 \leq n \leq N-1$, corrupted with additive white Gaussian noise. $\bar{N}$ was chosen equal to 256 . A Hamming window of 17 samples width was used in computing the STFT. It may be noted that in-band (bandpass) noise cannot be eliminated by the above procedure. To quantify the signal enhancement, we consider 100 realizations of the noisy signal, obtain their enhanced versions and compute the resulting SNR. Fig. 1 shows that there is an improvement in SNR because of the enhancement procedure (the dash-dot line shows the $45^{\circ}$ line for reference). The standard deviation in the output SNR is superposed on the plot as an error bar.

To show the utility of the enhancement scheme for $\mathrm{ZC}$ based IF estimation, let us consider the same phase signal. Fig. 2 shows the bias, variance and mean square error (all in $\mathrm{dB}$, obtained from 100 statistical realizations) for the proposed estimator using only $\mathrm{ZC}$ information (for the sake of illustration only) with and without enhancement (ENH). The improvement in performance due to the enhancement scheme is evident. For the same MSE, without enhancement, we require a high input SNR compared to the enhanced one. For high SNRs, roughly beyond $10 \mathrm{~dB}$, the performance of both approaches is the sarne. For the performance evaluation of the LC based IF estimation, we consider only the enhanced

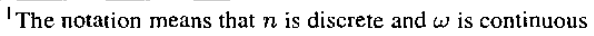



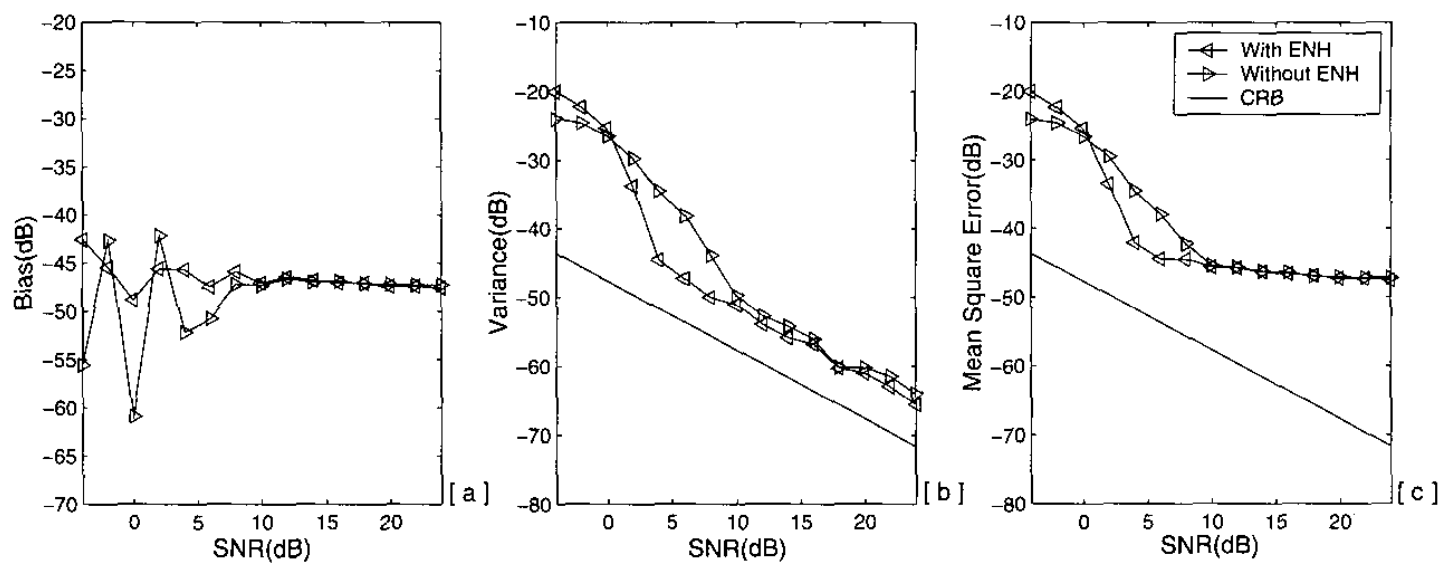

Fig. 2. Effect of enhancement on LPS based IF estimation - [a]Bias,[b]Variance,[c]MSE, all in dB, as a function of SNR(dB) at the center of the window.

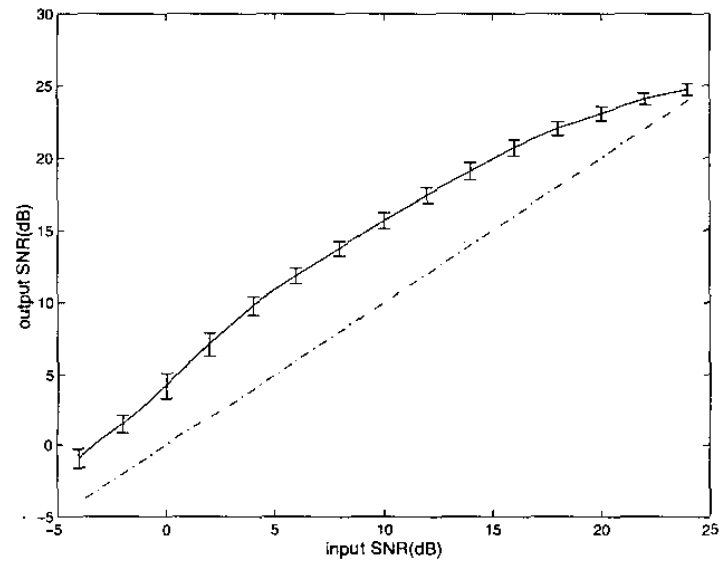

Fig. 1. Input-output SNR characteristics of the enhancement scheme (solid line) in comparison with the $45^{\circ}$ line for reference (dash-dot)

signal. For the sake of brevity, we omit ENH while mentioning the LC approaches with the understanding that it is always present. In the absence of any apriori knowledge about the rate of change of IF, we found that a wide-band STFT is suitable for enhancement; hence a 17-point Hamming window is used in all simulations.

\section{SIMULATION STUDIES}

In this section, we study the performance analysis of the proposed technique for a general composite IF, same as the one considered in Sec. 4.

10 levels, equispaced from 0 to 0.9 were used. Additive white Gaussian noise was added at different SNRs and for each SNR, 100 Monte-Carlo trials were run to obtain the statistics of the estimator. The variance of the estimate at the center of the window is shown against the level value with SNR as the parameter in Fig. 3. It can be clearly seen that as the level value increases, the variance of the estimator increases. This holds for all SNRs. It is also clear that for levels close to zero, the variance is more or less that given by the $\mathrm{ZC}$ based IF estimator.

The performance of different level-crossing based IF estimators as a function of SNR can also be studied from the same figure. It can be clearly seen that the $\mathrm{ZC}$ based IF estimator yields the least bias, variance and mean square error for a range of SNRs.

Different combinations of the level-crossing based IF estimates were tried and only three are reported here, namely mean, median and weighted mean. The statistics of the resulting estimator are shown in Fig. 4. The results are compared with those of a ZC based estimator. It is clear that the $\mathrm{ZC}$ estimator yields the best performance, followed by the median. Median of different levelcrossing estimates at any point outperforms their average because of the outlier rejection property of the median. Estimates obtained from high-level values often have large errors and are effectively suppressed by the median. A weighted average (weights $=\left[\begin{array}{lllll}1 & 1 & 1 & 1 & 1\end{array}\right.$ $110.80 .60 .4]$ ) yields an estimate that has a performance characteristic that lies between the $\mathrm{ZC}$ estimator and the simple average based estimator. As the weightage to higher-level crossing based estimates was increased, the error was found to increase. This is also intuitively satisfying.

Fig. 4 shows that the ZC based IF estimator lies close to the Cramer-Rao (CR) bound (discussed in next section) and is suboptimal in the sense that it does not exactly reach the bound. However, the bias, variance and mean square error are small enough to make the estimator relevant and useful in many practical applications. A comparative study with other methods proposed in literature will be reported separately [5].

\section{CRAMER-RAO BOUND}

We briefly outline the derivation for obtaining the CRB [6] for the proposed $\mathrm{IF}$ estimator. Consider a unity amplitude phase signal $s(t)=e^{j \phi(t)}$ in additive white Gaussian noise of energy density $N_{0}$. We assume that there is no modeling bias. The entries in the Fisher information matrix $J$ are $J_{\alpha, \alpha}=\frac{2 T}{N_{0}}, J_{\beta, \beta}=\frac{2 T^{3}}{3 N_{0}}, J_{\alpha, \beta}=$ 

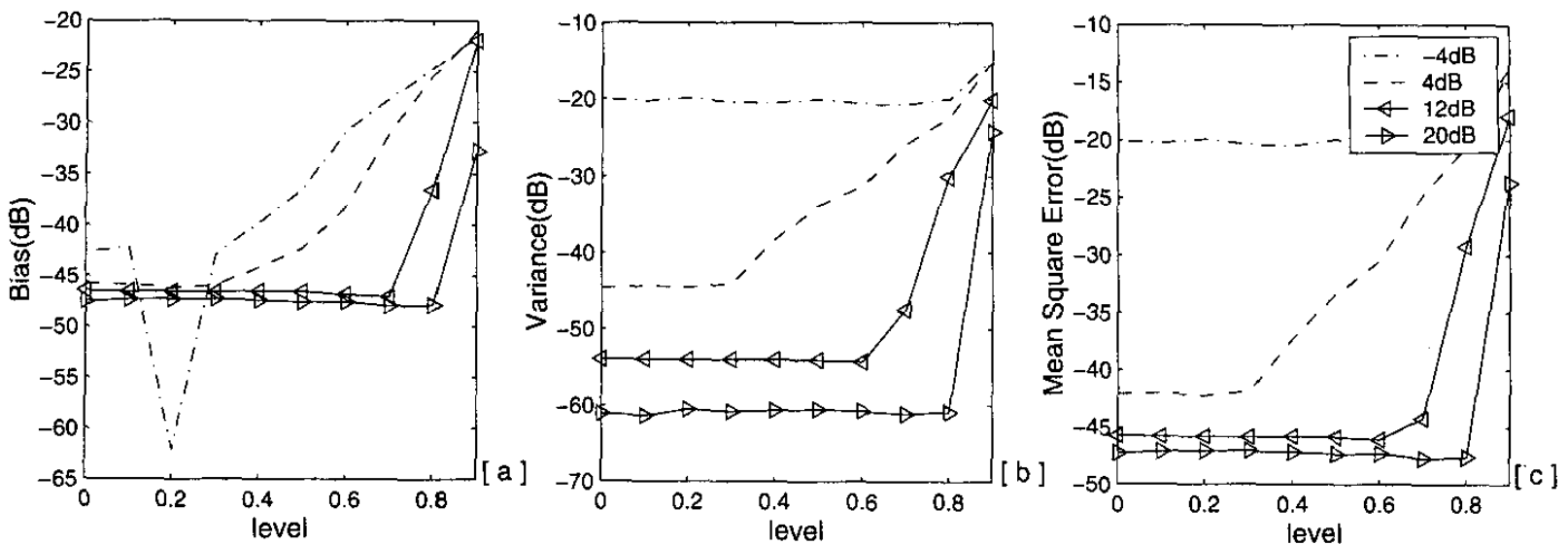

Fig. 3. [a] Bias(dB), [b] Variance(dB), [c] Mean Square Error(dB) Vs, level as a function of $S N R(d B)$
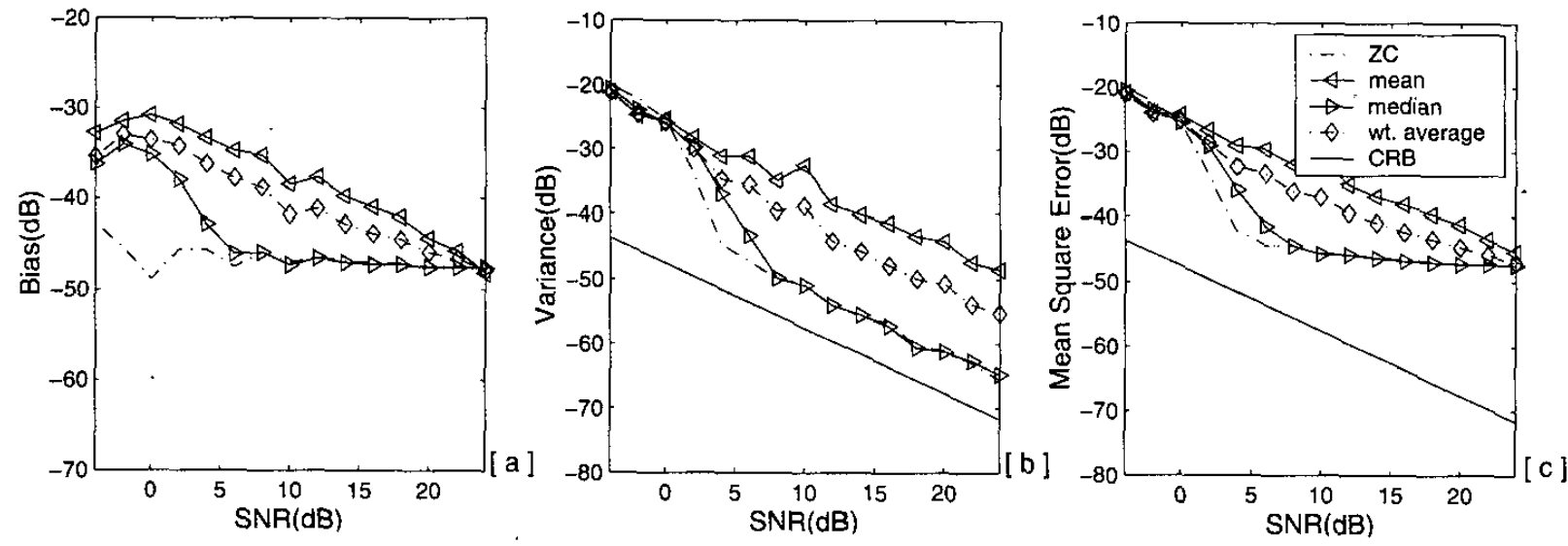

Fig. 4. [a] Bias(dB), [b] Variance(dB), [c] Mean Square Error(dB) as a function of SNR(dB), with CRB for reference.

$0, J_{\alpha, \gamma_{k}}=0, J_{\beta, \gamma_{k}}=\frac{-2 T^{2}}{\pi k N_{0}}, J_{\gamma_{k}, \gamma_{m}}=\frac{T}{N_{0}} \delta_{k, m}$. The CRBs for $\phi(t)$ and $f_{i}(t)$ are given by $C R B\{\phi(t)\}=v_{1} J^{-1} v_{1}^{T}, C R B\left\{f_{i}(t)\right\}=$ $\frac{1}{4 \pi^{2}} v_{2} J^{-1} v_{2}^{T}$, where where $v_{1}=\left[1 t \sin \left(\omega_{0} t\right) \sin \left(2 \omega_{0} t\right) \ldots\right.$ $\left.\sin \left(p \omega_{0} t\right)\right]$ and $v_{2}=\left[\begin{array}{llll}0 & 1 & \omega_{0} \cos \left(\omega_{0} t\right) & 2 \omega_{0} \cos \left(2 \omega_{0} t\right)\end{array} \ldots\right.$ $\left.p \omega_{0} \cos \left(p \omega_{0} t\right)\right]$.

\section{CONCLUSION}

In this paper, we introduced the concept of time-warping in the context of IF estimation. A multi-level crossing based approach along with a novel line plus sum of sines model has been proposed for IF estimation. The emphasis has been on estimating generalized IF. IF estimation in the presence of noise has been considered. To reduce the error in the resulting estimate, an enhancement procedure is suggested. It has been demonstrated through extensive simulations, that a zero-crossing instant based IF estimator exhibits least error compared to higher level-crossing based estimates.

\section{REFERENCES}

[1] T.V.Sreenivas and R.J.Niederjohn, "Zero-crossing Based Spectral Analysis and SVD Spectral Analysis for Formant Frequency Estimation in Noise", IEEE Trans. Sig. Proc., vol. 40, no. 2, Feb 1992.

[2] B.Kedem, "Time Series Analysis by Higher Order Crossings", IEEE Press, 1993.

[3] B.Boashash, "Estimating and Interpreting the Instantaneous Frequency of a Signal - Part-2: Algorithms and Applications", Proc. IEEE, vol. 80, no. 4, pp. 539-568, April 1992.

[4] M.Unser, "Sampling-50 Years After Shannon", Proc. IEEE, vol. 88, no.4, April 2000.

[5] S.Chandra Sekhar and T.V.Sreenivas, "Instantaneous Frequency Estimation Using Level-Crossing Information", manuscript under preparation

[6] S.M.Kay, Fundamentals of Statistical Signal Processing: Estimation Theory, Prentice Hall Englewood Cliffs, New Jersey, 1993. 